\title{
Detection of Merkel cell polyomavirus using whole exome sequencing data
}

Sandra Garcia-Mulero',2,3, Ferran Moratalla-Navarro', ${ }^{1,2}$, Soraya Curiel-Olmo4, Victor Moreno ${ }^{1,2,3}$, José Pedro Vaqué4,5, Rebeca Sanz-Pamplona ${ }^{1,2 *}$ and Josep María Piulats ${ }^{1,6 *}$

1. ONCOBELL Program, Bellvitge Biomedical Research Institute (IDIBELL). Hospitalet de Llobregat, Barcelona. Spain.

2. Biomarkers and Susceptibility Unit (UBS), Oncology Data Analytics Program (ODAP), Catalan Institute of Oncology (ICO). Hospitalet de Llobregat, Barcelona. Spain. Consortium for Biomedical Research in Epidemiology and Public Health (CIBERESP), Spain.

3. Department of Clinical Sciences, Faculty of Medicine and Health Sciences, University of Barcelona, Spain.

4. Infection, Immunity and Digestive pathology group. IDIVAL, Santander, Spain.

5. Molecular Biology Department. University of Cantabria, Santander, Spain.

6. Department of Medical Oncology, Catalan Institute of Oncology (ICO), Hospitalet de Llobregat, Barcelona, Spain. Consortium for Biomedical Research in Oncology (CIBERONC), Spain.

* co-corresponding authors

Correspondence to:

Josep María Piulats: jmpiulats@iconcologia.net

Rebeca Sanz-Pamplona: rebecasanz@iconcologia.net

Gran Vía de L'Hospitalet, 199-203

08908, L'Hospitalet de Llobregat, Barcelona. Spain

\begin{abstract}
Merkel cell carcinoma (MCC) is a highly malignant neuroendocrine tumor of the skin in which Merkel cell polyomavirus (MCV) DNA virus insertion can be detected in $75-89 \%$ of cases. Etiologic and phenotypic differences exist between MCC tumors with and without the inserted virus, thus it is important to distinguish between MCV+ MCC and MCV- MCC cases. Currently, MCV insertions in MCC genomes are detected using laboratory techniques. Here we report a freely available bioinformatics methodology to identify $\mathrm{MCV}+\mathrm{MCC}$ tumors using whole exome sequencing (WES) data. WES data could be also used to infer the virus insertion site into the tumor genome. Our method has been validated in a set of MCC samples previously characterized in the laboratory as MCV+ or MCV-, achieving 100\% sensitivity and 62,5\% specificity. Thus, with enough depth of sequencing, it is possible to use WES to the presence of MCV insertions in cancer samples.
\end{abstract}

Keywords: Sequencing, Merkel cell carcinoma, viral insertion, pipeline. 


\section{Introduction}

Merkel cell carcinoma (MCC) is highly malignant neuroendocrine tumor of the skin currently thought to arise from mechanoreceptor Merker cells [1]. MCC typically affects immunosuppressed individuals thus suggesting an infectious origin. Indeed, in 2008, a $5.4 \mathrm{kbp}$ polyomavirus DNA was found to be integrated in some MCC genomes in a clonal pattern [2]. Although MCC is rare, its incidence has tripled over the past two decades in the Unites States. This could be due for both the increasing prevalence of risk factors such as ultraviolet (UV) exposure or systemic immune suppression [3] or because of the improvement of diagnostic methods. Merkel cell polyomavirus (MCV) DNA virus can be detected in $75-89 \%$ of MCC. It is noteworthy that etiologic and phenotypic differences exist between MCC tumors with and without the inserted virus [4]. In this regard, recent work by independent laboratories has shown important genetic differences between $\mathrm{MCV}+$ and MCV- MCC tumors, the latter harboring higher mutational burdens with ultraviolet (UV) signatures [5-8]. Moreover, relevant clinical features are associated with this phenotype being the MCV- subtype the one showing a worst prognosis [9]. Also, from a therapeutic point of view, immunotherapy could play an important role in this tumor $[10,11]$. Thus, it is important to distinguish between MCV+ MCC and MCV- MCC. Normally, MCV insertions in MCC genomes were detected by PCR or using an anti-MCP antibody [9]. Here we show a 2-alignment-steps methodology to identify MCV+ tumors using WES data.

\section{Results and discussion}

\section{2-alignment-steps pipeline}

The following pipeline was set up using a positive control sample (WES data from a MCV+ MCC tumor analyzed with standard laboratory techniques): after quality control assessment using FastQC [12], WES raw data was pre-processed with TrimGalore [https://github.com/FelixKrueger/ TrimGalore] for adapters and bad quality reads removal. Then, reads were aligned against the human reference genome with Bowtie2 [13] using strict parameters to achieve a very sensitive local alignment. Next, unmapped reads (reads that did not map to human genome) were retrieved using SamTools [14]. Finally, these non-human reads were aligned to the NCBI Merkel cell polyomavirus reference genome (EU375803.1; isolate MCC350, complete genome). This second alignment was performed with BWA aligner [15]. If reads were found matching with MCV genome, the tumor was classified as $\mathrm{MCV}+($ Figure 1). In detail description of the pipeline is found in the Materials and Methods section. The script used to make this analysis is freely available at Github repository 


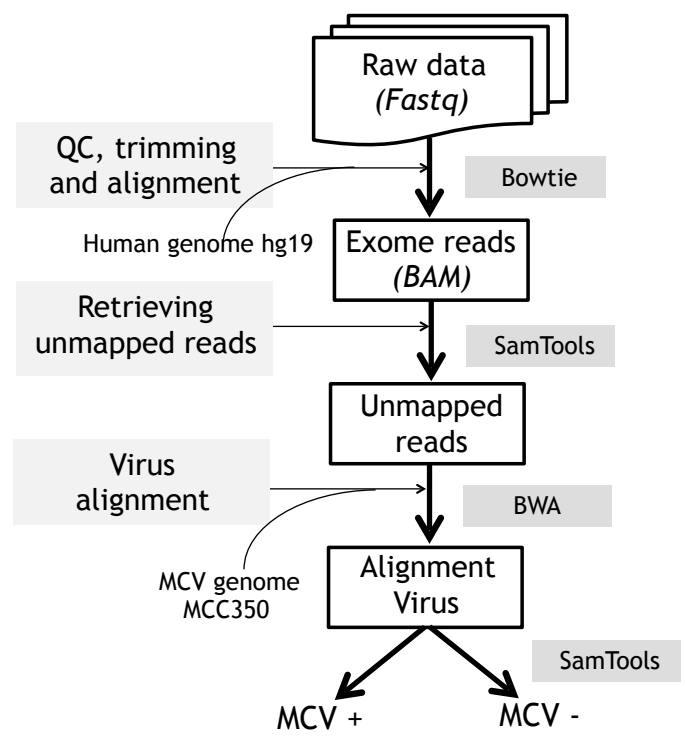

Figure 1. Pipeline for Merkel virus insertion detection using WES data.

(https:/github.com/odap-ubs/merkel_virus). We had previously assessed that there was no similarities between the MCV genome and any sequence in the Homo sapiens genome. The MCV genome is about $5 \mathrm{~Kb}$ long, and splits of $75 \mathrm{bp}$ (a total of 70 ) were submitted to the online tool BLAST [16]. No homology was found. Per sample coverage was calculated as twice the read length multiplied by total number of reads and divided by total genome length.

\section{MCV insertion in MCC patients}

We interrogated sequencing data for a total of $15 \mathrm{MCC}$ primary tumors and their normal paired samples, previously characterized in the laboratory for MCV insertions (seven $\mathrm{MCV}+$, and eight MCV-) [5]. Our method was able to detect all seven MCC positive for MCV (ranged from 2 to 44 mapped reads). Supplementary Figure 1 shows the MCV+ MCC aligned reads in the Integrative Genome Viewer (IGV) [17]. Interestingly, there was a tendency towards detecting more aligned reads in the first 2,500 bp of the $\mathrm{MCV}$. As expected, none of the normal samples were found to present MCV insertions. Strikingly, three out of eight previously classified as MCV- MCC were found to have at least one read mapping into MCV genome whereas the remaining five had zero mapped reads. Sensitivity and specificity were $100 \%$ and $62.5 \%$, respectively. However, due to the extremely low number of reads in false positive samples, better values of specificity would be obtained if a sample was catalogued as positive only when at least two MCV reads were detected. It was also interesting to note that there was a correlation between the sequencing coverage and the number of mapped reads. This may indicate that when depth of sequencing increases also increases the probability of finding an inserted viral genome (Table 1). 
bioRxiv preprint doi: https://doi org/10.1101/2020.04.27.063214: this version posted April 28,2020 . The copyright holder for this preprint (which was not certified by peer review) is the author/funder, who has granted bioRxiv a license to display the preprint in perpetuity. It is made available under aCC-BY-NC-ND 4.0 International license.

Table 1. Alignment results.

\begin{tabular}{|c|c|c|c|c|}
\hline SAMPLE & TISSUE & MCP & $\mathbf{N}^{\mathbf{0}}$ MAPPED READS & COVERAGE \\
\hline 48120427 & FF & + & 44 & 3.519 \\
\hline 48120428 & FF & + & 34 & 2.704 \\
\hline 48121209 & FF & + & 16 & 2.543 \\
\hline 48120987 & FF & + & 15 & 2.286 \\
\hline 48140221 & FF & + & 7 & 2.090 \\
\hline 48090369 & FF & + & 4 & 2.467 \\
\hline 48130247 & FFPE & + & 2 & 1.794 \\
\hline 48130206 & FFPE & - & 2 & 1.304 \\
\hline 48120431 & FF & - & 1 & 3.213 \\
\hline 48141029 & FFPE & - & 1 & 1.784 \\
\hline 48141028 & FFPE & - & 0 & 1.869 \\
\hline 48130208 & FFPE & - & 0 & 1.364 \\
\hline 48130207 & FFPE & - & 0 & 1.691 \\
\hline 48121576 & FF & - & 0 & 2.520 \\
\hline 48120426 & FF & - & 0 & 3.192 \\
\hline
\end{tabular}

Since interrogated samples were a mixture of fresh frozen (FF) and formalin-fixed paraffinembedded (FFPE) preserved tumors, we wondered if our method performed well in both types of samples. Figure 2 suggest that our pipeline is more robust when NGS was performed in FF samples. This is not surprising since sequencing performs better in FF tissues. However, only one out of seven MCV+ MCC were FFPE. Moreover, the MCV+ FFPE sample was the one with the lower sequencing depth so a coverage bias rather than a tissue preservation effect cannot be excluded. Thus, more samples need be assessed in order to settle this topic.

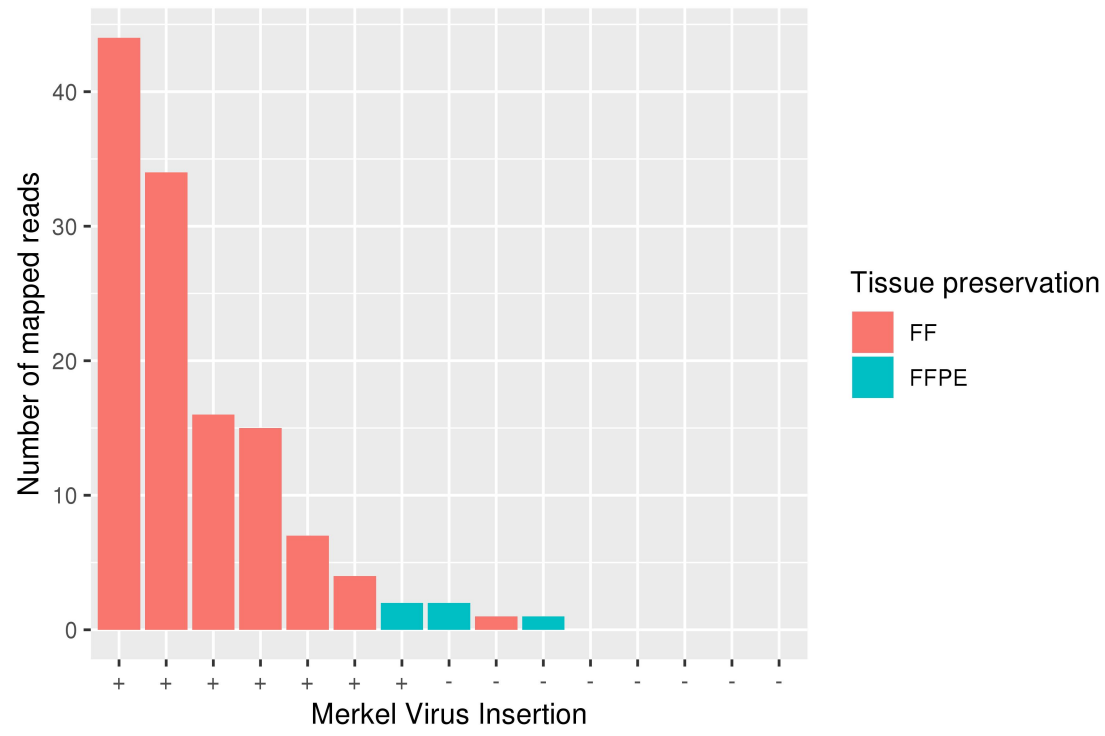

Figure 2. Barplot representing the number of mapped reads by sample. Fresh frozen (FF) samples were represented in orange and formalin fixed paraffin embedded (FFPE) in green. In the $\mathrm{x}$ axis, positive (+) and negative (-) MCC tumors were indicated. 


\section{MCV site of insertion}

Finally, paired-end WES data was used to try to infer the virus insertion site into the tumor genome. After applying this strategy in all positive samples, only one of them (48121209) showed softclipped reads whose mates were mapped on chr19:48,445,990. Interestingly, this region was into GRWD1 gene intronic region (Supplementary Figure 2A). This is a P53 regulator whose loss of function has been previously associated with tumorigenesis [18]. The fact that this is a highly covered region might indicate that our method failed to detect insertion positions in poor covered regions. Thus, whole-genome sequencing should be a better technique to identify not only MCV+ tumors but also the virus insertion site. Supplementary Figure 2B showed how soft-clipped reads also mapped against the MCV genome.

Apart from MCC, MCV insertions have been found in small-cell lung cancer in non-smokers [19]. However, this is a controversial result since other study has reported no MCV insertions in this cancer [20]. Moreover, MCV DNA fragments could be detected in the lower respiratory tract when a high-sensitive PCR assay was used, thus false positives could not be excluded [21]. Therefore, the 2-alignment-steps methodology was applied on non-smokers lung adenocarcinoma data sets; 25 RNA-seq samples [22] and 30 WES samples [23], but no MCV+ tumor was found.

\section{Conclusions}

In conclusion, here we present an easy method to detect MCV insertions as a bystander product of exome sequencing. Since, human gene exons DNA are enriched in WES, MCV will be most likely detected in MCC if its genome is inserted in exome regions or when high sequencing depth is obtained and MCV reads are detected as off-target reads. We cannot rule out the possibility that samples were contaminated with viral DNA, but the lack of positive results in paired normal samples sequenced with similar coverage was reassuring. Thus, with enough depth of sequencing, it is possible to apply the pipeline described here to take advantage of WES experiments and assess the presence of MCV insertions in any dataset of interest.

\section{Materials and Methods}

\section{Patients and samples}

WES data from a total of 15 MCC patients and their normal paired samples were used for the analysis. Samples were either formalin fixed paraffin embedded (FFPE) or freshly frozen (FF). For clinical characteristics and genomic analysis, refer to González-Vela et al. study [5]. For validation purposes, data from the European Nucleotide Archive (ENA) public repository was downloaded; 25 
RNA-seq samples from series SRP090460 [22], and 30 WES samples from series SRP022932 [23] were used.

\section{Identification of MCV+ tumors using WES}

First, raw data was evaluated for quality control with FastQC. Next, samples were trimmed if necessary with TrimGalore (v0.4.0) and aligned against the human reference genome build hg19/ GRCh37, previously indexed, using Bowtie 2.0 (v2.2.5) - alignment 1-. From this first alignment, unmapped reads are selected with Samtools (v1.3.1). Function Samtools view, with -f and F options, was used to select and filter the desired reads. The online utility "Decoding SAM flags" from the Broad Institute (https://broadinstitute.github.io/picard/explain-flags.html) was used to decide the filtering criteria. The selection criteria filters: i) unmapped reads whose mate are mapped (-f 4 -F 264), ii) mapped reads whose mates is unmapped (-f 8 -F 260), iii) both unpaired reads (-f 12 -F 256). The three outputs are merged and next, those retrieved unmapped reads are aligned against MCV genome (5,381 bp length, downloaded from the NCBI - GenBank accession number EU375803.1; Merkel cell polyomavirus isolate MCC350, complete genome-) with BWA (v0.7.15) -alignment 2-. Finally, the number of reads aligned to virus is calculated, and an output is generated with the following information: Sample ID, MCV+/- status, and read counts in case of $\mathrm{MCV}+$.

\section{Virus insertion site}

After the first alignment against the human reference genome, unmapped and discordant reads were kept to be aligned against the MCV genome. Of these, reads mapped to the MCV genome whose mates were mapped to the human genome were used to get information of the proximal insertion site (Supplementary Figure 3). On the other hand, a visual inspection of the bam files using IGV tool was done in order to find the exact site of insertion.

Author Contributions: RSP and JMP designed the study, conceived the experiments and wrote the article. SGM and FMN carried out the sequencing analyses. SCO and JPV performed wet lab experiments. VM provides biostatistics expertise. VM and JPV helped to draft the manuscript. All authors critically reviewed and had final approval of the article.

Funding: Agency for Management of University and Research Grants (AGAUR) of the Catalan Government grant 2017SGR723; Instituto de Salud Carlos III, co-funded by FEDER funds -a way to build Europe- grants PI14-00613, PI17-00092.

Acknowledgments: We thank Josipa Bilic for critical reading of the manuscript and English language assistance. We thank Spanish Association Against Cancer (AECC) Scientific Foundation. Conflicts of Interest: Victor Moreno is consultant to Bioiberica S.A.U. and Grupo Ferrer S.A., received research funds from Universal DX and is co-investigator in grants with Aniling. Josep 
M Piulats is consultant for Roche-Genentech, Bristol Myers Squibb, Merck Sharp \& Dohme, Merck-Serono, Janssen, Astellas, VCN-Biotech, and BeiGene; Josep M Piulats has received research grants from Bristol Myers Squibb, Merck Shart \& Dohme, Merck Serono, Janssen, and Astra Zeneca.

\section{References}

1. Harms PW, Harms KL, Moore PS et al. The biology and treatment of Merkel cell carcinoma: current understanding and research priorities. Nat Rev Clin Oncol 2018; 15(12):763-776.

2. Feng H, Shuda M, Chang Y, Moore PS. Clonal integration of a polyomavirus in human Merkel cell carcinoma. Science 2008; 319(5866):1096-1100.

3. Fitzgerald TL, Dennis S, Kachare SD et al. Dramatic Increase in the Incidence and Mortality from Merkel Cell Carcinoma in the United States. Am Surg 2015; 81(8):802-806.

4. Shuda M, Arora R, Kwun HJ et al. Human Merkel cell polyomavirus infection I. MCV T antigen expression in Merkel cell carcinoma, lymphoid tissues and lymphoid tumors. Int. J. Cancer 2009; 125(6):1243-1249.

5. González-Vela MDC, Curiel-Olmo S, Derdak S et al. Shared Oncogenic Pathways Implicated in Both Virus-Positive and UV-Induced Merkel Cell Carcinomas. J. Invest. Dermatol. 2017; 137(1):197-206.

6. Harms PW, Vats P, Verhaegen ME et al. The Distinctive Mutational Spectra of PolyomavirusNegative Merkel Cell Carcinoma. Cancer Res. 2015; 75(18):3720-3727.

7. Wong SQ, Waldeck K, Vergara IA et al. UV-Associated Mutations Underlie the Etiology of MCV-Negative Merkel Cell Carcinomas. Cancer Res. 2015; 75(24):5228-5234.

8. Goh G, Walradt T, Markarov V et al. Mutational landscape of MCPyV-positive and MCPyVnegative Merkel cell carcinomas with implications for immunotherapy. Oncotarget 2016; 7(3):3403-3415.

9. Moshiri AS, Doumani R, Yelistratova L et al. Polyomavirus-Negative Merkel Cell Carcinoma: A More Aggressive Subtype Based on Analysis of 282 Cases Using Multimodal Tumor Virus Detection. J. Invest. Dermatol. 2017; 137(4):819-827.

10. Colunga A, Pulliam T, Nghiem P. Merkel Cell Carcinoma in the Age of Immunotherapy: Facts and Hopes. Clin. Cancer Res. 2018; 24(9):2035-2043.

11. Nghiem P, Bhatia S, Lipson EJ et al. Durable Tumor Regression and Overall Survival in Patients With Advanced Merkel Cell Carcinoma Receiving Pembrolizumab as First-Line Therapy. J. Clin. Oncol. 2019; 37(9):693-702.

12. Andrews S. (2010). FastQC: a quality control tool for high throughput sequence data. Available online at: http://www.bioinformatics.babraham.ac.uk/projects/fastqc 
13. Langmead B, Salzberg S. Fast gapped-read alignment with Bowtie 2. Nature Methods. 2012, 9:357-359.

14. Li H.*, Handsaker B.*, Wysoker A., Fennell T., Ruan J., Homer N., Marth G., Abecasis G., Durbin R. and 1000 Genome Project Data Processing Subgroup (2009) The Sequence alignment/ map (SAM) format and SAMtools. Bioinformatics, 25, 2078-9.

15. Heng Li, Richard Durbin, Fast and accurate short read alignment with Burrows-Wheeler transform, Bioinformatics, Volume 25, Issue 14, 15 July 2009, Pages 1754-1760, https://doi.org/ 10.1093/bioinformatics/btp324.

16. Madden T. The BLAST Sequence Analysis Tool. 2002 Oct 9 [Updated 2003 Aug 13]. In: McEntyre J, Ostell J, editors. The NCBI Handbook [Internet]. Bethesda (MD): National Center for Biotechnology Information (US); 2002-. Chapter 16. Available from: http://www.ncbi.nlm.nih.gov/ books/NBK21097/.

17. James T. Robinson, Helga Thorvaldsdóttir, Wendy Winckler, Mitchell Guttman, Eric S. Lander, Gad Getz, Jill P. Mesirov. Integrative Genomics Viewer. Nature Biotechnology 29, 24-26 (2011).

18. Kayama K, Watanabe S, Takafuji T et al. GRWD1 negatively regulates p53 via the RPL11MDM2 pathway and promotes tumorigenesis. EMBO Rep. 2017; 18(1):123-137.

19. Hashida Y, Imajoh M, Daibata M. Integrated and mutated forms of Merkel cell polyomavirus in non-small cell lung cancer. Br. J. Cancer 2013; 108(12):2624.

20. Busam KJ, Jungbluth AA, Rekthman N et al. Merkel Cell Polyomavirus Expression in Merkel Cell Carcinomas and Its Absence in Combined Tumors and Pulmonary Neuroendocrine Carcinomas. The American Journal of Surgical Pathology 2009; 33(9):1378.

21. Babakir-Mina M, Ciccozzi M, Lo Presti A et al. Identification of Merkel cell polyomavirus in the lower respiratory tract of Italian patients. J. Med. Virol. 2010; 82(3):505-509.

22. Sun, Z., Wang, L., Eckloff, B.W. et al. Conserved recurrent gene mutations correlate with pathway deregulation and clinical outcomes of lung adenocarcinoma in never-smokers. BMC Med Genomics 7, 486 (2014). https://doi.org/10.1186/1755-8794-7-32.

23. Ahn, J.W., Kim, H.S., Yoon, J. et al. Identification of somatic mutations in EGFR/KRAS/ALKnegative lung adenocarcinoma in never-smokers. Genome Med 6, 18 (2014). https://doi.org/ $10.1186 / \mathrm{gm} 535$. 


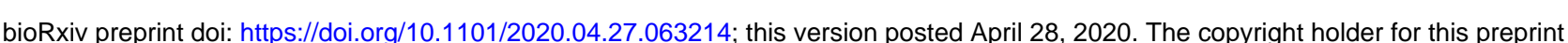
(which was not certified by peer review) is the author/funder, who has granted bioRxiv a license to display the preprint in perpetuity. It is made available under aCC-BY-NC-ND 4.0 International license.

\section{Supplementary Materials}

Supplementary Figure 1. MCV+ MCC alignment IGV visualization. Upper line represented the $\mathrm{MCV}$ genome length. Each line corresponds to the seven MCV+ MCC tumor samples.

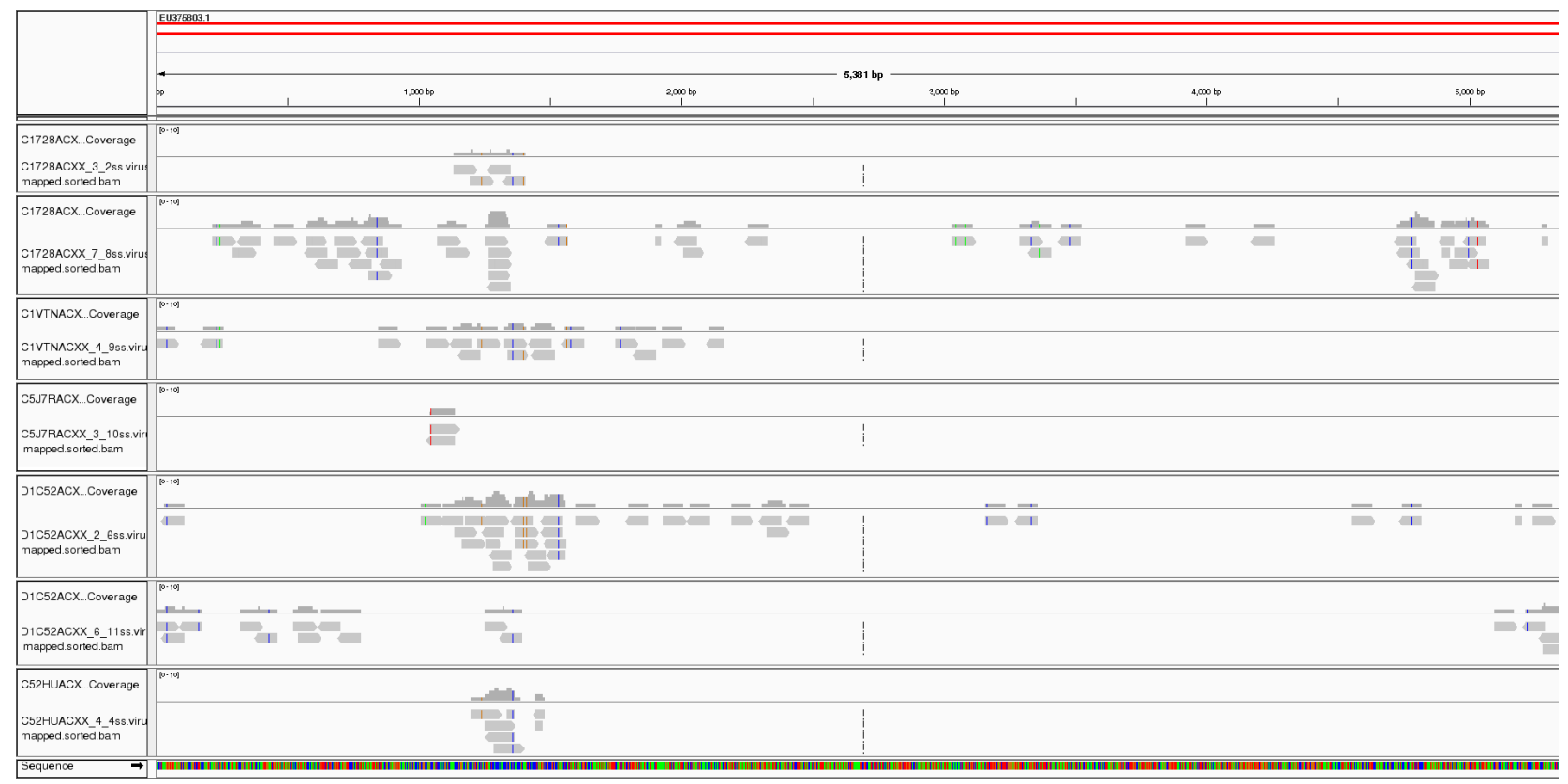


bioRxiv preprint doi: https://doi org/10.1101/2020.04 27.063214 this version posted April 28,2020 . The copyright holder for this preprint (which was not certified by peer review) is the author/funder, who has granted bioRxiv a license to display the preprint in perpetuity. It is made available under aCC-BY-NC-ND 4.0 International license.

Supplementary Figure 2. MCV insertion site in MCC sample 48121209. A. Breakpoint regions in GRWD1 gene (chromosomal coordinates chr19:48,445,990). Several soft-clipped reads in human alignment in the central part of the image, indicating the exact breakpoint in this sample. B. Breakpoint regions in MCV. The rainbowed part of reads (soft-clipping representation) indicates the site of the reads unable to map into MCV genome.

A

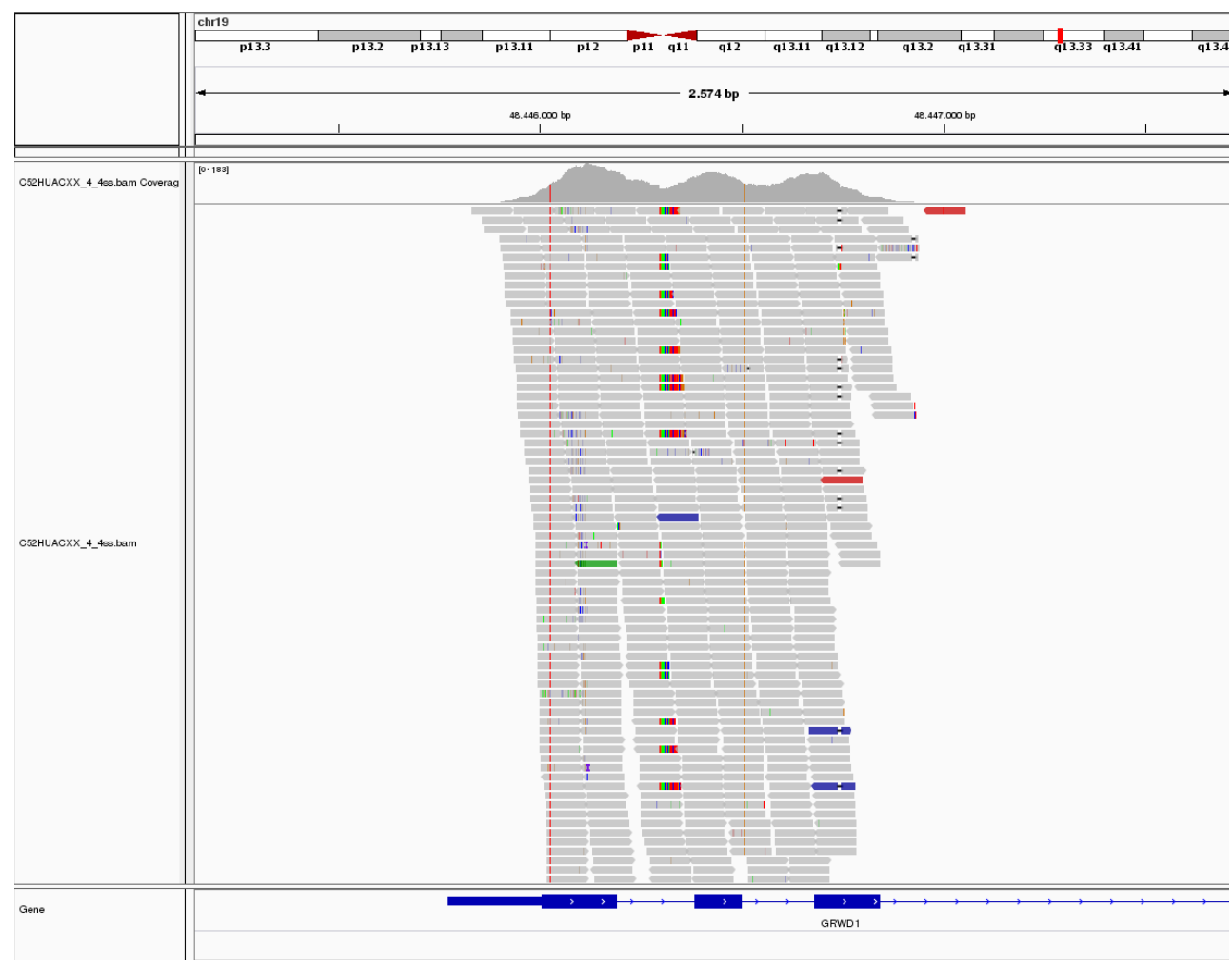

B

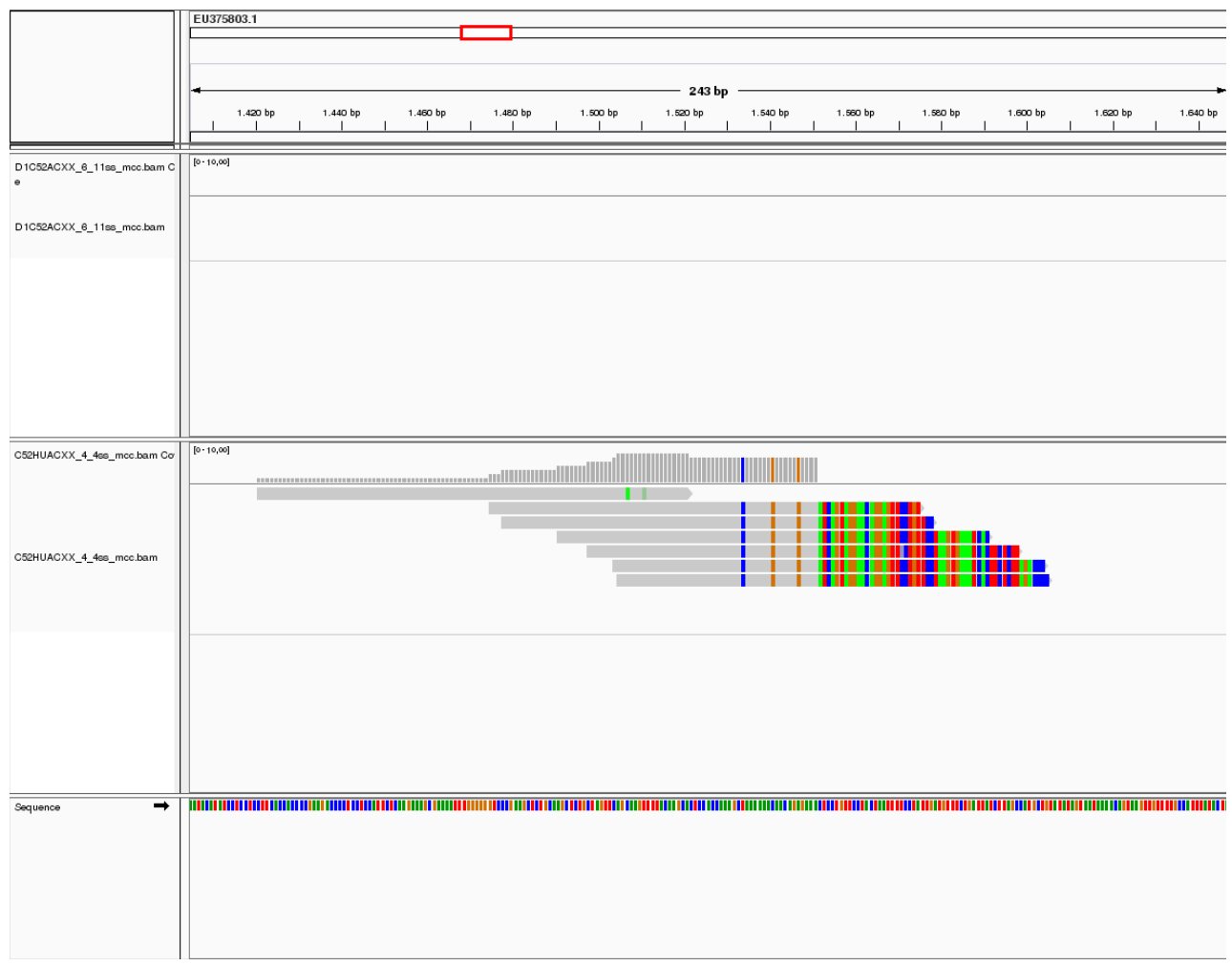


bioRxiv preprint doi: https://doi.org/10.1101/2020.04 27.063214; this version posted April 28, 2020. The copyright holder for this preprint (which was not certified by peer review) is the author/funder, who has granted bioRxiv a license to display the preprint in perpetuity. It is made available under aCC-BY-NC-ND 4.0 International license.

Supplementary Figure 3. MCV insertion site into human genome pipeline. Bam files aligned into human and into MCV genome virus previously obtained were used. Discordant mapped reads of MCV alignment whose mates were mapped into human were used to narrow the possible insertion site to a few hundred of base pairs. Next, IGV was used to inspect these regions in search for soft-clipped reads both in human and virus alignment that confirmed the exact insertion site.

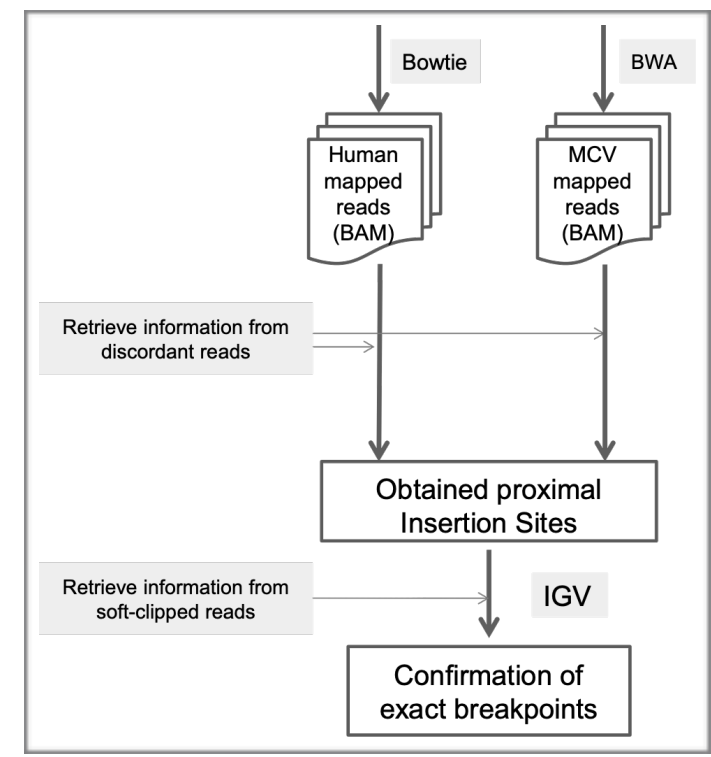

